\title{
Glucocorticoid Suppresses Dendritic Spine Development Mediated by Down-Regulation of Caldesmon Expression
}

\author{
Daisuke Tanokashira, ${ }^{1 \star}$ Tsuyoshi Morita, ${ }^{1 \star}$ Ken'ichiro Hayashi, ${ }^{1 \star}$ Taira Mayanagi, ${ }^{1,3}$ Kentaro Fukumoto, $^{1,3,4}$ \\ Yoshiko Kubota, ${ }^{3}$ Toshihide Yamashita, ${ }^{2}$ and Kenji Sobue ${ }^{1,3}$ \\ Departments of ${ }^{1}$ Neuroscience and ${ }^{2}$ Molecular Neuroscience, Graduate School of Medicine, Osaka University, Suita, Osaka 565-0871, Japan, ${ }^{3}$ Department of \\ Neuroscience, Institute for Biomedical Sciences, Iwate Medical University, Yahaba-cho, Shiwa-gun, Iwate 028-3694, Japan, and ${ }^{4}$ Department of \\ Neuropsychiatry, School of Medicine, Iwate Medical University, Morioka, Iwate, 020-8505, Japan
}

Glucocorticoids (GCs) mediate the effects of stress to cause structural plasticity in brain regions such as the hippocampus, including simplification of dendrites and shrinkage of dendritic spines. However, the molecular mechanics linking stress and GCs to these effects remain largely unclear. Here, we demonstrated that corticosterone (CORT) reduces the expression levels of caldesmon (CaD), causing dendritic spines to become vulnerable. $\mathrm{CaD}$ regulates cell motility by modulating the actin-myosin system and actin filament stability. In cultured rat hippocampal neurons, $\mathrm{CaD}$ localized to dendritic spines by binding to filamentous actin (F-actin), and CaD expression levels increased during spine development. $\mathrm{CaD}$ stabilized the F-actin dynamics in spines, thereby enlarging the spine heads, whereas $\mathrm{CaD}$ knockdown decreased the spine-head size via destabilization of the F-actin dynamics. CaD was also required for chemical LTP-induced actin stabilization. The CaD expression levels were markedly decreased by exposure to CORT mediated by suppression of serum response factor-dependent transcription. High CORT levels reduced both the spine-head size and F-actin stability similarly to CaD knockdown, and overexpressing $\mathrm{CaD}$ abolished the detrimental effect of CORT on dendritic spine development. These results indicate that CaD enlarges the spine-head size by stabilizing F-actin dynamics, and that $\mathrm{CaD}$ is a critical target in the GC-induced detrimental effects on dendritic spine development.

\section{Introduction}

Glucocorticoid (GC) hormones are principal stress mediators released from the adrenal gland in response to stressful events. Prolonged stress and chronic GC exposure produce abnormal behaviors in experimental animals and an increased risk of psychiatric disorders in humans (McEwen, 1999, 2005; Phillips et al., 2005; Becker et al., 2007). In animals, maternal stress during pregnancy increases GC levels not only in the mother but also in the fetus, leading to structural alterations in the developing brain and abnormal behaviors in adult offspring (Weinstock, 2008). In humans, prenatal stress is associated with increased anxiety in adolescence and a greater incidence of psychiatric disorders in adulthood (McEwen, 2005; Phillips et al., 2005; Becker et al., 2007). Antenatal GC therapy in humans has been shown to reduce both the convolution index and surface area of the cortex (Modi et al., 2001). Exposure to repeated stress

Received May 16, 2012; revised Aug. 1, 2012; accepted Aug. 21, 2012.

Author contributions: T. Morita, K.H., T.Y., and K.S. designed research;D.T., T. Morita, K.H., T. Mayanagi, K.F., and Y.K. performed research; D.T., T. Morita, K.H., and T. Mayanagi analyzed data; T. Morita and K.S. wrote the paper.

This work was supported by Grants-in-aid for Scientific Research 20240038 from the Japan Society for the Promotion of Science (to K.S.) and 23110510 from the Ministry of Education, Culture, Sports, Science and Technology (MEXT) (to K.S).

${ }^{*}$ D. T., T. Morita, and K. H. contributed equally to this work.

The authors declare no competing financial interests.

Correspondence should be addressed to Dr. Kenji Sobue, Department of Neuroscience, Institute for Biomedical

Sciences, Iwate Medical University, 2-1-1 Nishitokuta, Yahaba-cho, Shiwa-gun, Iwate 028-3694, Japan. E-mail: ksobue@iwate-med.ac.jp.

DOI:10.1523/JNEUROSCI.2380-12.2012

Copyright $\odot 2012$ the authors $\quad 0270-6474 / 12 / 3214583-09 \$ 15.00 / 0$ and excessive GCs adversely affects neuronal architecture, causing dendritic branches to atrophy and reducing the density of dendritic spines and changing spine morphology (Watanabe et al., 1992; Magariños et al., 1996; Wellman, 2001; Radley et al., 2004, 2006; Liston and Gan, 2011). Thus, repeated exposure to stress-induced GCs results in alterations of neuronal architecture related to psychiatric disorders, but the precise molecular and cellular mechanisms of these alterations have been largely unclear.

Most excitatory synapses are located in the dendritic spines, which are small actin-rich protrusions that change shape from filopodia to stubby or mushroom-shaped spines during the course of neuron development (von Bohlen Und Halbach, 2009). Spine morphology and density are of importance as structural foundation in cognitive function and behavior (Dumitriu et al., 2010; Bloss et al., 2011). Actin filaments, a central component of dendritic spines, regulate the spine morphology, dynamics, and function (Hotulainen and Hoogenraad, 2010). In long-term potentiation (LTP), which is considered a cellular event for memory formation, the spine heads enlarge and synaptic connections are strengthened. Actin filament formation is tightly associated with the establishment of LTP (Lisman, 2003; Hotulainen and Hoogenraad, 2010). Thus, actin dynamics play an important role in synaptic development and plasticity. Although chronic stress and GC exposure are reported to impair synaptic development and plasticity (Radley et al., 2006; Liston and Gan, 2011), the molecular mechanisms of their association with actin dynamics remain poorly understood. 

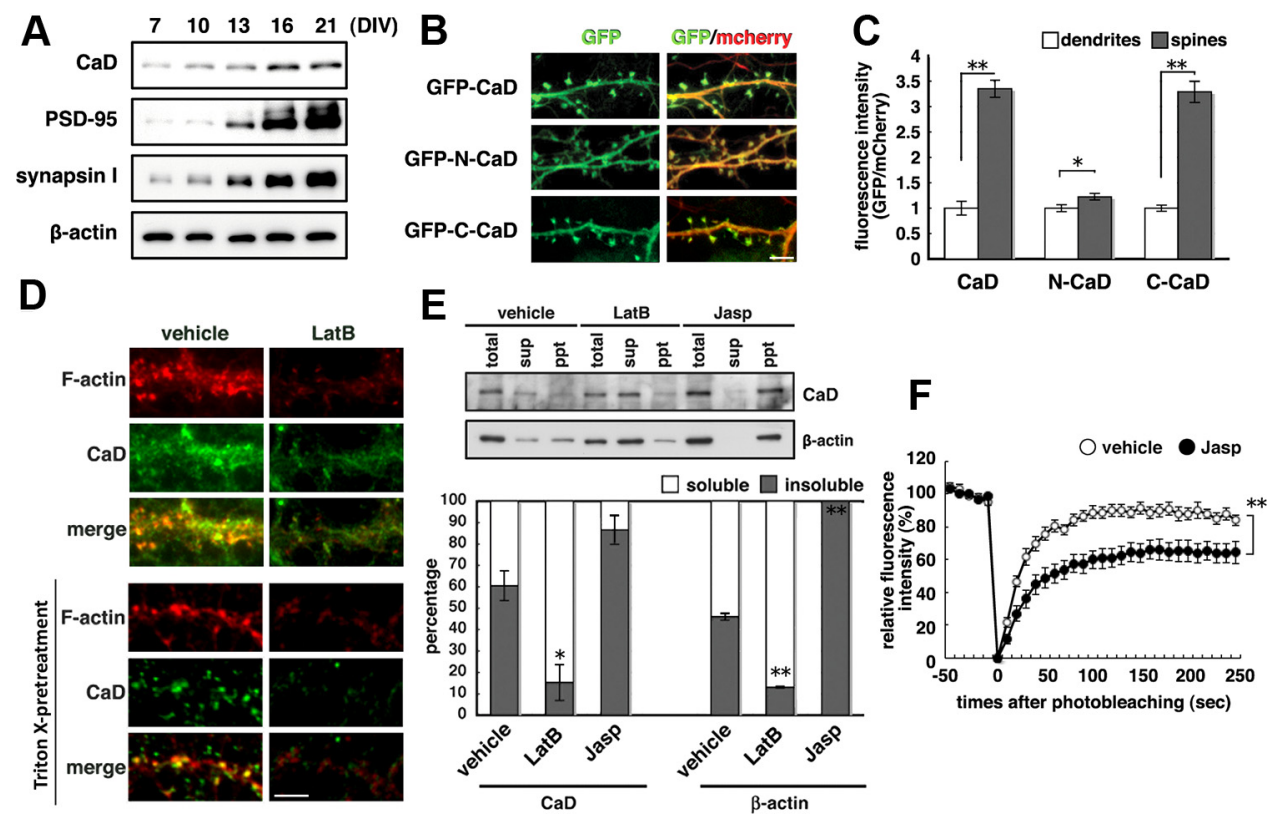

Figure 1. CaD expression profile and localization in developing hippocampal neurons. $A$, CaD, PSD-95, and synapsin I protein expression profiles. B, GFP-CaD, GFP-N-CaD, or GFP-C-CaD was exogenously expressed in neurons, using mCherry protein as a cell volume marker (scale bar, $5 \mu \mathrm{m}$ ). GFP and mCherry fluorescence intensities were measured in dendritic shafts and spines, respectively. C, The graph shows GFP intensities corrected for mCherry intensities. CaD associates with F-actin ( ${ }^{*} p<0.05,{ }^{* *} p<0.01$ ). D, Hippocampal neurons treated with DMSO (vehicle) or LatB for $3 \mathrm{~h}$ were incubated with (bottom) or without (top) $1 \%$ Triton X-100, then fixed and stained with anti-CaD antibody (green) and phalloidin (F-actin, red) (scale bar, $5 \mu \mathrm{m}$ ). $\boldsymbol{E}$, Neurons were treated with DMSO (vehicle), LatB, or Jasp, and then lysed with a solution containing Triton X-100. The lysates were separated into Triton X-soluble and -insoluble fractions by centrifugation. Western blotting was performed using equal load volumes of the fractions (top), and the $\mathrm{CaD}$ and $\beta$-actin proteins were quantified by densitometry (bottom). Data are means $\pm S E$ of values from three independent experiments $\left({ }^{*} p<0.05,{ }^{* *} p<0.01\right)$. $\boldsymbol{F}$, GFP-CaD fluorescence recovery over time after photobleaching, measured in neurons treated with DMSO (vehicle) or jasplakinolide (Jasp). Data are means $\pm \mathrm{SE}$ of values from 10 cells from three independent experiments $\left({ }^{* *} p<0.01\right)$.

Caldesmon $(\mathrm{CaD})$ is a ubiquitous actin-linked regulatory protein that binds to and stabilizes actin filaments (Sobue and Sellers, 1991; Mayanagi and Sobue, 2011). GCs affect expression level of $\mathrm{CaD}$, which negatively controls the radial migration of neural progenitor cells by regulating actin-myosin interactions, leading to transiently retarded neocortical development (Fukumoto et al., 2009). CaD also regulates axon extension by inhibiting myosin II function (Morita et al., 2012). Thus, the CaD-mediated regulation of actin dynamics is important for brain development. However, the role of $\mathrm{CaD}$ in spine development is unclear. In this study we demonstrated that $\mathrm{CaD}$ stabilizes actin filaments and increases the head size of dendritic spines in hippocampal neurons, while GCs decrease $\mathrm{CaD}$ expression levels and cause the detrimental effects on spine development.

\section{Materials and Methods}

Antibodies. This study used the following purchased antibodies: antiPSD-95 (MA1-045, Affinity BioReagents), anti- $\beta$-actin (A5441, Sigma), anti-SRF (sc-335, Santa Cruz Biotechnology), anti-GAPDH (FL-335, Santa Cruz Biotechnology), anti-GFP (A11120 and A11122, Invitrogen), and anti-DsRed (632496, Clontech). Anti-CaD and anti-synapsin1 antibodies were produced in rabbits.

Cell culture. Since the medium generally used to culture hippocampal neurons includes a B27 supplement containing an undisclosed concentration of CORT, we used CORT-free B27 supplement commissioned from Invitrogen and added CORT to the medium to reach defined concentrations. Hippocampal neurons were prepared from rats on embryonic day 18.5. Dispersed neurons were plated on poly-L-lysine-coated coverslips and cultured in glial-conditioned MEM containing $1 \mathrm{~mm}$ sodium pyruvate, $0.6 \%(\mathrm{~W} / \mathrm{V}) \mathrm{D}$-glucose, and either $2 \%$ standard B 27 supplement (Invitrogen) or 2\% CORT-free B27 supplement with the indicated concentrations of CORT added. After 1 week, one-half of the medium was changed to neurobasal (Invitrogen) medium containing 0.5 mM L-glutamine (Sigma) with either 2\% standard B27 supplement or $2 \%$
CORT-free B27 supplement with the indicated concentrations of CORT added, and the neurons were cultured for another 2 weeks. All experiments using the cultured rat hippocampal neurons were performed during 21-24 d in vitro (DIV). All animal experiments were performed in accordance with guidelines from the Osaka University School of Medicine and the Iwate Medical University.

Foot-shock stress. Male Wistar rats (9-10 weeks old) (Japan SLC) were housed under controlled $12 \mathrm{~h} / 12 \mathrm{~h}$ light/dark (lights on at 7:00 A.M.) and temperature $\left(23 \pm 2^{\circ} \mathrm{C}\right)$ conditions. Rats were randomly allocated to stress or control groups, and rats in the stress group were subjected to a single session of foot shocks. Foot-shock stress was applied in a footshock box with a grid floor connected to a shock generator (Med Associates) controlled by FreezeFrame software (Actimetrics). During this session, the rats received four uncontrollable, inescapable foot shocks (current intensity, 1.0 mA; duration, $1.0 \mathrm{~s}$ at random intervals of 60-90 s). After the session, the rat remained in the chamber for $60 \mathrm{~s}$ and was then returned to its home cage.

Determination of CORT concentrations. To avoid the influence of timerelated CORT fluctuations, all experiments using live rats were performed between 10:30 A.M. and 11:00 A.M. Rats were anesthetized and decapitated, and blood was collected from the heart. The blood plasma was collected by centrifugation. At the same time, the brain was quickly removed, and cerebral tissue samples were individually homogenized in ice-cold PBS using a Potter-Elvehjem homogenizer and an ultrasound sonicator. Tissue homogenates were centrifuged and the supernatants were collected. Blood plasma and brain extracts were immediately stored at $-80^{\circ} \mathrm{C}$. CORT concentrations in these samples were determined with the Corticosterone EIA Kit (YK240, Yanaihara), which directly determines CORT concentrations using specially formulated sample diluents that displace CORT-binding globulin (CBG).

Expression plasmids and transfection. The coding regions for human $l-\mathrm{CaD}$ and its $\mathrm{N}$ terminus (1-263 aa) and $\mathrm{C}$ terminus (264-558 aa) and for human $\beta$-actin were amplified by PCR and subcloned into the highly efficient mammalian expression plasmid pCAGGS. EGFP, mCherry, or the myc-tag sequence was fused to the $5^{\prime}$ end of the coding sequences. 
A
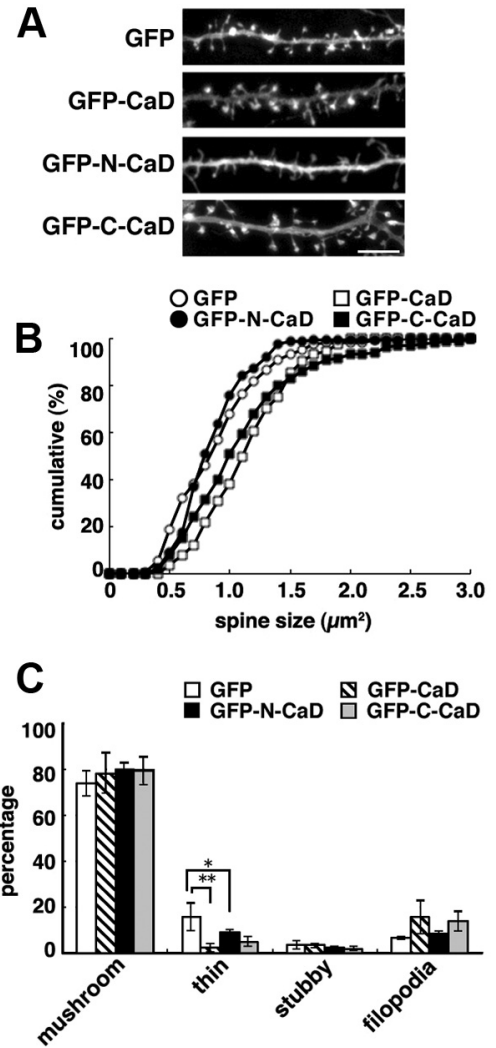

D
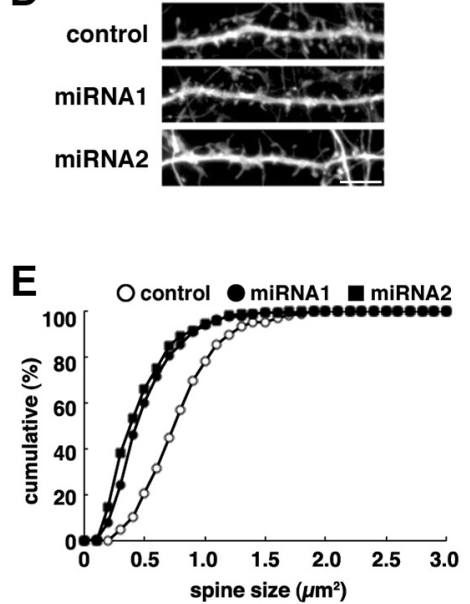

$\mathbf{F}$

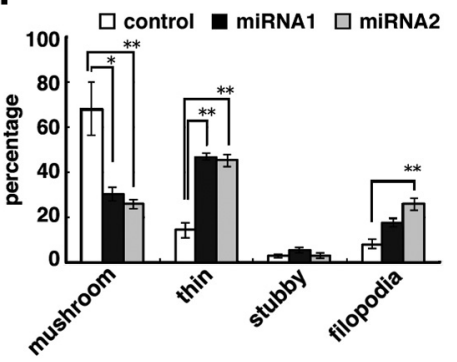

Figure 2. CaD regulates spine size in hippocampal neurons. $A$, Spine morphology in neurons transfected with GFP, GFP-CaD, GFP-N-CaD, or GFP-C-CaD (scale bar, $10 \mu \mathrm{m}$ ), and graphs of spine sizes and their cumulative distributions (B), and classification of spine morphology (C). D, Spine morphology in neurons transfected with control miRNA, CaD miRNA1, or CaD miRNA2 (scale bar, 10 $\mu \mathrm{m})$, and graphs of spine sizes and their cumulative distributions $(\boldsymbol{E})$, and classification of spine morphology $(\boldsymbol{F})$.

The mCherry-LifeAct and miRNA plasmids were constructed as previously reported (target sequences: CaD miRNA1, GAGATGTATCTGGCAAGCGGA; CaD miRNA2, CTGGAGCAATATACCAATGCA) (Fukumoto et al., 2009; Morita et al., 2012). Hippocampal neurons were transfected by the calcium phosphate method as described previously (Jiang and Chen, 2006). Transfection was performed at 7 DIV (for miRNA) or 19 DIV (for CaD-overexpression).

Imaging. Neurons were cultured on coverslips, fixed using $4 \%$ paraformaldehyde, incubated with a primary antibody, and then incubated with the appropriate Alexa-labeled secondary antibody. To visualize filamentous actin (F-actin), Alexa 568-phalloidin (Invitrogen) was added in the secondary antibody solution. Stained neurons were observed under a BIOREVO BZ-9000 (Keyence) fluorescence microscope with an $\times 100$ oil-immersion lens. Living neurons were cultured in a glassbottom dish with Tyrode's solution ( $119 \mathrm{~mm} \mathrm{NaCl}, 2.5 \mathrm{~mm} \mathrm{KCl}, 25 \mathrm{~mm}$ HEPES, $30 \mathrm{~mm}$ glucose, $2 \mathrm{~mm} \mathrm{CaCl}_{2}$, and $2 \mathrm{~mm} \mathrm{MgCl}_{2}, \mathrm{pH} 7.4$ ) and observed under an LSM5 PASCAL laser-scanning microscope (Carl Zeiss) with an $\times 63$ oil-immersion lens.

To monitor actin turnover in dendritic spines, neurons transfected with GFP and mCherry- $\beta$-actin were analyzed by FRAP assay as previously described (Star et al., 2002). A high-intensity $543 \mathrm{~nm}$ He-Ne laser was used to bleach the mCherry fluorescence in single spines, and the fluorescence recovery was observed with a low-power laser every $10 \mathrm{~s}$. GFP fluorescence was simultaneously detected with a $488 \mathrm{~nm}$ argon laser to monitor spine volume change. Actin turnover in the spine was evaluated by the fluorescence recovery of mCherry- $\beta$-actin, corrected for background and GFP intensity to avoid the influence of spine volume changes during observation. The GFP-CaD dynamics in spines were also analyzed by FRAP. Neurons were transfected with GFP-CaD and mCherry. The GFP-CaD fluorescence in a single spine was bleached and the fluorescence recovery was observed every $10 \mathrm{~s}$. The GFP-CaD intensity was corrected for background and mCherry intensity. The FRAP curve was fit to the equation: $F(t)=1-f_{s}-f_{f}$ * $\exp ^{(-t / \lambda)}$ in the previous report (Star et al., 2002), where $f_{s}, f_{f}, t$, and $\lambda$ are the stable fraction, dynamic fraction, time (second), and time constant, respectively. The time constant during fluorescence recovery was analyzed according to the equation for the first $60 \mathrm{~s}$.

To monitor F-actin dynamics in dendritic spines, neurons were transfected with GFP and mCherry-LifeAct. Fluorescence was detected every $10 \mathrm{~s}$. F-actin dynamics were evaluated by the average change rate per $10 \mathrm{~s}$ of mCherry intensity, corrected for background and EGFP intensity to avoid the influence of spine volume change during observation. Actin-depolymerizing drug, latrunculin B (LatB, $5 \mu \mathrm{M})$, and actin-stabilizing drug jasplakinolide (Jasp, $100 \mathrm{~nm}$ ) were used in actin dynamics-targeted experiments.

Chemical LTP. Chemical LTP (cLTP) was induced in cultured hippocampal neurons as previously described (Otmakhov et al., 2004). Neurons were incubated for $15 \mathrm{~min}$ in Tyrode's solution containing $6 \mu \mathrm{M} 2$-chloroadenosine. The solution was replaced by $\mathrm{MgCl}_{2}-$ free Tyrode's solution containing $100 \mu \mathrm{M}$ picrotoxin, $50 \mu \mathrm{M}$ forskolin, and $0.1 \mu \mathrm{M}$ rolipram, and the neurons were incubated for another $15 \mathrm{~min}$. After cLTP was induced, the solution was washed out with normal Tyrode's solution. Neurons were observed $3 \mathrm{~h}$ after cLTP induction.

Triton X-100-insoluble cytoskeletons. Neurons were treated with BRB buffer $(10 \mathrm{~mm}$ PIPES pH 7.4, 3 mм NaCl, $100 \mathrm{~mm} \mathrm{KCl,} 3.5 \mathrm{~mm}$ $\mathrm{MgCl}_{2}$, and $1.25 \mathrm{~mm}$ EGTA) containing $1 \%$ Triton X-100 and protease inhibitor cocktail (Takara) for $2 \mathrm{~min}$ and were then washed with Triton X-100-free BRB buffer. The neurons were fixed and stained with anti-CaD antibody and phalloidin. For sedimentation assays, neurons were lysed with BRB buffer containing Triton X-100, and the lysate was separated by ultracentrifugation at $100,000 \times g$ for $2 \mathrm{~h}$. The supernatant and pellet were subjected to Western blotting as the Triton X-soluble and -insoluble fractions, respectively.

Quantitative real-time PCR. Total RNA was extracted from neurons, and cDNA was synthesized using the High-Capacity cDNA Reverse Transcription Kit (Applied Biosystems); mRNA levels were quantified by real-time PCR (RT-PCR) using SYBR GreenER qPCR SuperMix (Invitrogen) and were normalized to GAPDH mRNA expression. The respective primers were designed and synthesized by Sigma as follows: Gapdh-F (forward): CTCCCATTCTTCCACCTTTGATG, Gapdh-R (reverse): CCACCACCCTGTTGCTGTAG; Cald1-F: ATGTGGGAGAAAGGG AGTGTG, Cald1-R: CTTGGGAGCGGGTGACTTG, Cald1-Fibrotype-F: CTTGCTGGGTTGCTTAAAGG, Cald1-HeLa-type-F: CAGCTGCGGACATGCTTAG， Cald1-common-R: CTGTCACCTGTCCCA AGGAT; $\beta$-actin-F: CGTTGACATCCGTAAAGACCTC, $\beta$-actin-R: ATAGAGCCACCAATCCACACAG; PSD-95-F: CAGTGAGACCGACGACATTGG, PSD-95-R: ATGATGATGGGACGAGCATAGTG.

Reporter assays. The reporter constructs for the fibroblast-type promoter region of the rat $C A L D 1$ gene were described previously (Fukumoto et al., 2009). The cells were lysed with Passive Lysis buffer (Promega), and the luciferase and $\beta$-galactosidase activities were measured using the luciferase assay system (Promega) and luminescent $\beta$-galactosidase detection kit II (Clontech), respectively.

Data quantification and statistics. Data from Western analysis were quantified by densitometry using ImageJ software. Data from synapse imaging were quantified using MetaMorph (Molecular Devices), BZ-II analysis application (Keyence; for fixed-cell imaging) or LSM5 PASCAL (Carl Zeiss; for live-cell imaging). All experiments were performed at 

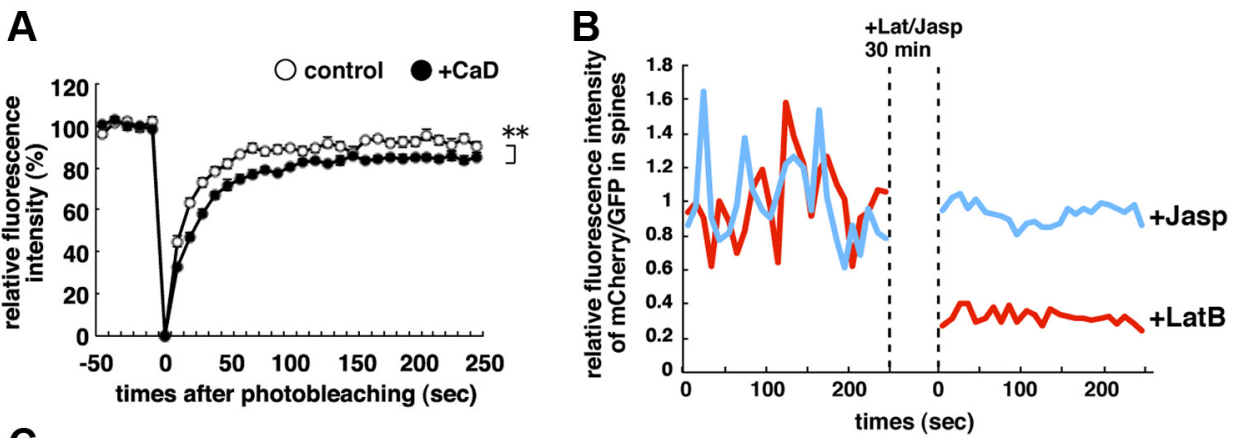

C
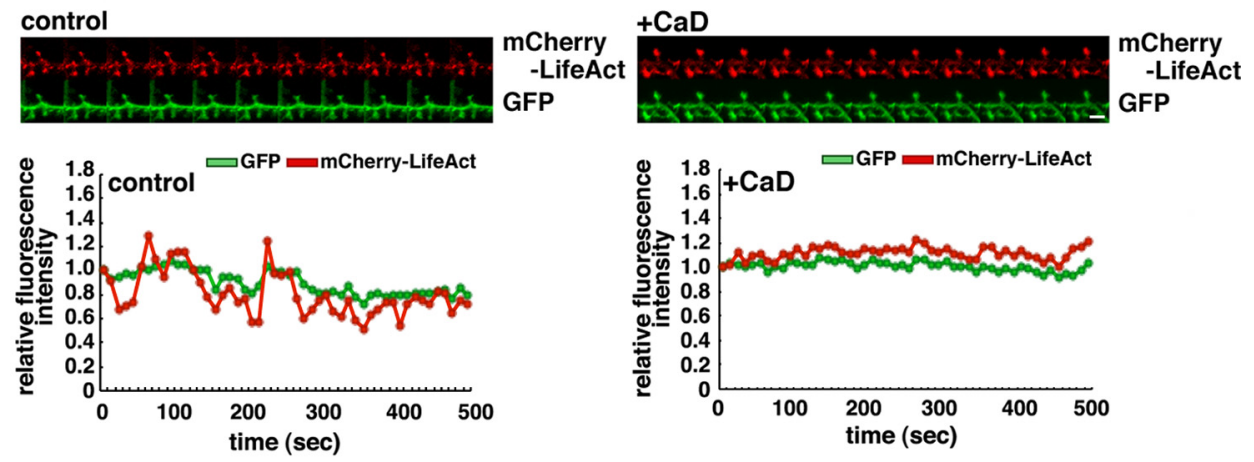

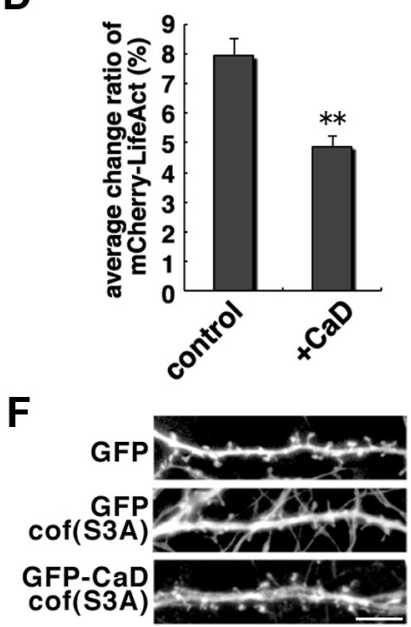

E before
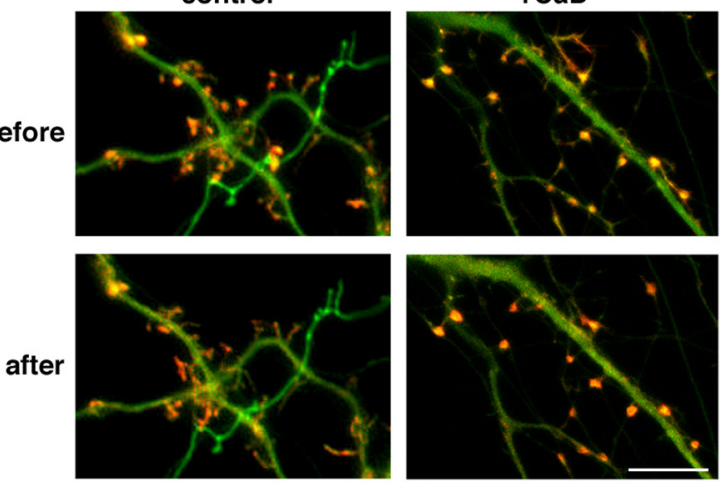

H

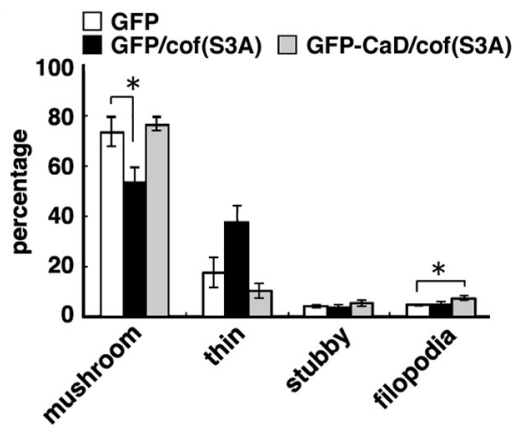

Figure 3. CaD effect on actin dynamics. $A$, The fluorescence recovery of mCherry- $\beta$-actin over time after photobleaching, measured in neurons transfected with a mock vector (control) or myc-tagged-CaD $(+\mathrm{CaD})$. Data are means \pm SE of values from 10 cells $\left({ }^{* *} p<0.01\right) \cdot \boldsymbol{B}$, Hippocampal neurons were transfected with GFP and mCherry-LifeAct to visualize spine volume changes and F-actin dynamics. The neurons were treated with Jasp or LatB for $30 \mathrm{~min}$ in the middle of the observation period, and changes in the $\mathrm{mCherry}$ and GFP fluorescence intensities in the spines were measured. The graph shows F-actin dynamics, corrected for the spine volume change (mCherry/GFP). F-actin dynamics were suppressed at high Jasp or low LatB levels; these agents elicit F-actin stabilization and depolymerization, respectively. C, Neurons were transfected with GFP, mCherry-LifeAct, and either a mock vector (control) or myc-CaD (+ CaD). Changes in fluorescence intensities in spines, observed every $10 \mathrm{~s}$ (top) (scale bar, $2 \mu \mathrm{m}$ ), are shown in the graph (bottom). Compared with GFP intensity changes, the mCherry intensity fluctuated dramatically in control neurons, whereas both the GFP and mCherry intensities were relatively stable in CaD-transfected neurons. D, Spine F-actin dynamics, observed with mCherry-LifeAct. The graph shows change rates of $m$ Cherry-LifeAct fluorescence intensity in spines. Data are means \pm SE of values from at least 18 spines $\left({ }^{* *} p<0.01\right)$. E, Neurons were transfected with GFP and $m$ Cherry-LifeAct to visualize the spine shape and the F-actin activity, and F-actin accumulation in spines was observed before or after LatB treatment. Scale bar, $10 \mu \mathrm{m}$. $\boldsymbol{F}$, Spine morphology in neurons transfected (Figure legend continues.) 


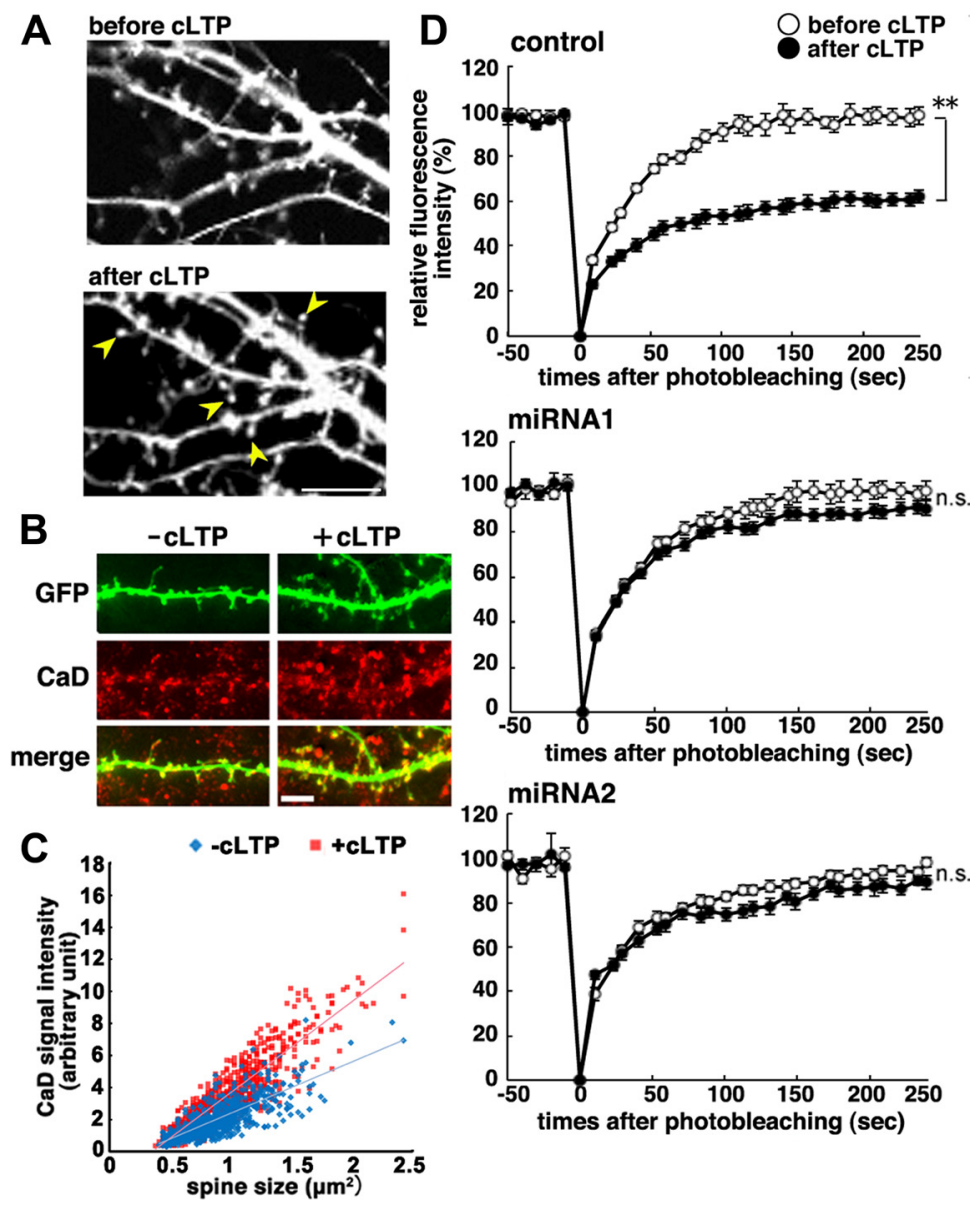

Figure 4. $\quad C a D$ is necessary for chemical LTP to induce F-actin stabilization in spines. A, Spine morphology before and after CLTP; yellow arrowheads indicate enlarged spines after CLTP (scale bar, $10 \mu \mathrm{m}$ ). $\boldsymbol{B}$, CaD accumulation in enlarged spines after CLTP (scale bar, $5 \mu \mathrm{m}$ ). $C$, Scatter plot of spine sizes versus $C a D$ intensities in each spine in neurons with (red square) or without CLTP (blue diamond). Data are obtained from at least 727 spines $(p<0.01)$. $\boldsymbol{D}$, Fluorescence recovery of $\mathrm{mCherry}-\beta$-actin over time after photobleaching, in hippocampal neurons transfected with control miRNA, CaD miRNA1, or CaD miRNA2. FRAP assays were performed before and after CLTP. Data are means \pm SE of values from at least 10 cells $\left({ }^{* *} p<0.01\right)$.

least three times independently. Statistical significance was evaluated by classical Student's $t$ test, paired $t$ test, or one-way ANOVA.

\section{Results}

$\mathrm{CaD}$ accumulates in spines

$\mathrm{CaD}$ levels increased during development of rat hippocampal neurons in culture and reached a peak at $16-21 \mathrm{~d}$ in vitro (DIV), concurrent with dramatic increases in the postsynaptic protein PSD-95 and the presynaptic protein synapsin I (Fig. 1A). GFPtagged full-length $\mathrm{CaD}$ (GFP-CaD) exogenously expressed in the neurons appeared to accumulate in spines (Fig. $1 B$ ). The $\mathrm{CaD}$ (GFP-C-CaD) C-terminal fragment, which includes actinbinding domains, was also localized to spines, while the $\mathrm{N}$-terminal fragment (GFP-N-CaD), which lacks actin-binding ability, was dispersed through both dendrites and spines (Fig. $1 B, C)$. These results suggest that $\mathrm{CaD}$ accumulates in spines by associating with $\mathrm{F}$-actin via its actin-binding domains. Consistent with this scenario, endogenous $\mathrm{CaD}$ was localized in the

\section{$\leftarrow$}

(Figure legend continued.) with GFP, GFP-CaD, and/or cof(S3A) (scale bar, $10 \mu \mathrm{m}$ ); the graph shows spine sizes and their cumulative distributions $(\boldsymbol{G})$ and classification of spine morphology $(\boldsymbol{H})$. Dendritic protrusions in the neurons were classified by morphology: mushroom, thin, stubby, or filopodial. Data are means \pm SE of values from at least 147 spines. spines and was resistant to Triton X-100 treatment (Fig. 1D, left). The Triton $\mathrm{X}-100$ resistance was abolished by the F-actin-depolymerizing agent latrunculin B (LatB) (Fig. $1 D$, right). In sedimentation assays, $\sim 60 \%$ of the $\mathrm{CaD}$ protein cosedimented with $\beta$-actin in the Triton X-100insoluble fraction, and LatB treatment shifted their localization to the soluble fraction (Fig. 1E). After treatment with the actin-polymerizing agent jasplakinolide (Jasp), $>80 \%$ of the $\mathrm{CaD}$ and $\beta$-actin cosedimented in the insoluble fraction (Fig. 1E). Fluorescence recovery after photobleaching (FRAP) assay also revealed that Jasp treatment reduced the GFP-CaD turnover rate in spines (Fig. $1 F)$. These data demonstrate that $\mathrm{CaD}$ is actively accumulated into spines through its association with F-actin.

\section{$\mathrm{CaD}$ enlarges the spine heads by stabilizing actin filaments}

To determine the function of $\mathrm{CaD}$ in spine dynamics, we overexpressed the $\mathrm{CaD}$ fragments in neurons. While GFP-CaD or GFP$\mathrm{C}-\mathrm{CaD}$ induced larger spine heads than GFP did alone, GFP-N-CaD did not (Fig. $2 A-C)$. We also depleted endogenous $\mathrm{CaD}$ using plasmid-type miRNA expression vectors, which were validated in previous studies (Fukumoto et al., 2009; Morita et al., 2012). Depleting endogenous CaD markedly reduced the spine-head size and changed the spine shape from mushroom to thin or filopodial (Fig. 2D-F). FRAP assay using mCherry- $\beta$-actin revealed that exogenous $\mathrm{CaD}$ significantly reduced the actin turnover rate within spines (Fig. 3A). The time constants (inverse of the turnover rate) for actin turnover were $19.3 \pm 1.1 \mathrm{~s}$ in GFP-transfected neurons versus $24.6 \pm 0.9 \mathrm{~s}$ in GFP-CaD-transfected neurons $(p<0.01)$. These data indicated that F-actin was stabilized. We also measured F-actin dynamics using mCherry-LifeAct, which selectively binds to F-actin and demonstrated real-time changes in the amount of F-actin within spines (Fig. 3B). The dendritic spines exhibit spontaneous motility as previously reported (Fischer et al., 1998, 2000; Korkotian and Segal, 2001; Star et al., 2002), and the dynamic motility is crucially based on the actin cytoskeleton (Fischer et al., 1998; Star et al., 2002). In control neurons, the amount of F-actin in spines varied dramatically compared with the spine volume traced by GFP (Fig. 3C). F-actin localization in CaD-transfected spines was more stable than that in control spines (Fig. 3C,D). This localization was resistant to LatB treatment (Fig. $3 E$ ), implicating that $\mathrm{CaD}$ stabilizes F-actin turnover in spines. Cofilin is considered to be a main factor for F-actin depolymerization and severing in spines (Lisman, 2003; Sarmiere and Bamburg, 2004). Indeed, cof(3A) (constitutively active cofilin) reduced the spine-head size (Fig. $3 F, G$ ). Interestingly, GFP$\mathrm{CaD}$ protected $\mathrm{F}$-actin from $\operatorname{cof}(3 \mathrm{~A})$ attack and thus promoted mushroom-shaped spines (Fig. $3 F-H$ ). Together, these results indicate that $\mathrm{CaD}$ stabilizes actin filaments in spines, leading to larger spine heads. 
CaD is necessary for chemical LTP to induce F-actin stabilization in spines Actin filament stabilization is tightly linked to LTP-induced spine-head enlargement (Lisman, 2003; Hotulainen and Hoogenraad). In cultured hippocampal neurons, cLTP triggered to enlarge spine heads (Fig. 4A) and to accumulate endogenous $\mathrm{CaD}$ into the enlarged spine heads (Fig. $4 B, C$ ), suggesting that accumulated $\mathrm{CaD}$ contributes to stabilizing actin filament in the spines. FRAP assay confirmed that CLTP-induced spine head enlargement was associated with F-actin stabilization (Fig. $4 A, D$ ). In CaD-knockdown neurons, however, cLTP treatment did not stabilize F-actin (Fig. $4 D$ ). These results strongly suggest that $\mathrm{CaD}$ is critical for cLTP-induced F-actin stabilization in spine head enlargement.

CORT decreases $\mathrm{CaD}$ expression levels We previously identified that $\mathrm{CaD}$ is one of the main downstream molecules in the GC-induced detrimental effects on the radial migration of neural progenitor cells during neocortical development (Fukumoto et al., 2009). Numerous studies have reported stress-induced increase in GC concentrations in the blood plasma or serum. However, GC concentration in the brain under nonstressed and stressed conditions remained unclear. Under conventional culture conditions without serum, neurons are, therefore, cultured in medium containing GC at nonstressed blood concentrations. To examine how GCs affect dendritic spine formation in cultured hippocampal neurons, we first examined the concentration of corticosterone (CORT), the principal GC in many species including rodents, in rat blood plasma and brain extracts after foot-shock stress. The CORT concentration increased markedly after stress, from $77.9 \pm 30.0 \mathrm{nM}$ to $1577.8 \pm 191.6 \mathrm{nM}(p<$ $0.01)$ in the blood plasma and from $16.7 \pm$

$0.5 \mathrm{nM}$ to $275.0 \pm 36.0 \mathrm{nM}(p<0.01)$ in the brain (Fig. $5 A)$. To culture hippocampal neurons with these CORT concentrations, our culture medium was prepared using CORT-free B27 supplement, with CORT added to defined concentrations. Importantly, the $\mathrm{CaD}$ levels decreased markedly according to the CORT dosage (Fig. $5 B-E$ ), and the F-actin was simultaneously decreased in spines (Fig. $5 D$, bottom). Thus, these results suggest that chronic CORT treatment decreases the localization of F-actin as well as $\mathrm{CaD}$ in spines, leading to the reduction of spine head size.

We previously reported that the $\mathrm{CaD}$ gene encodes multiple isoforms, and that the fibro- and HeLa-type promoter regions, which function independently, regulate their transcription (Yano et al., 1994). In hippocampal neurons, CORT treatment suppressed the fibro-type isoforms more severely than the HeLa-type isoforms (Fig. 6A). Reporter assays showed that CORT reduced the $\mathrm{CaD}$ fibro-type promoter activity (Fig. $6 B$ ). The CaD pro-
A
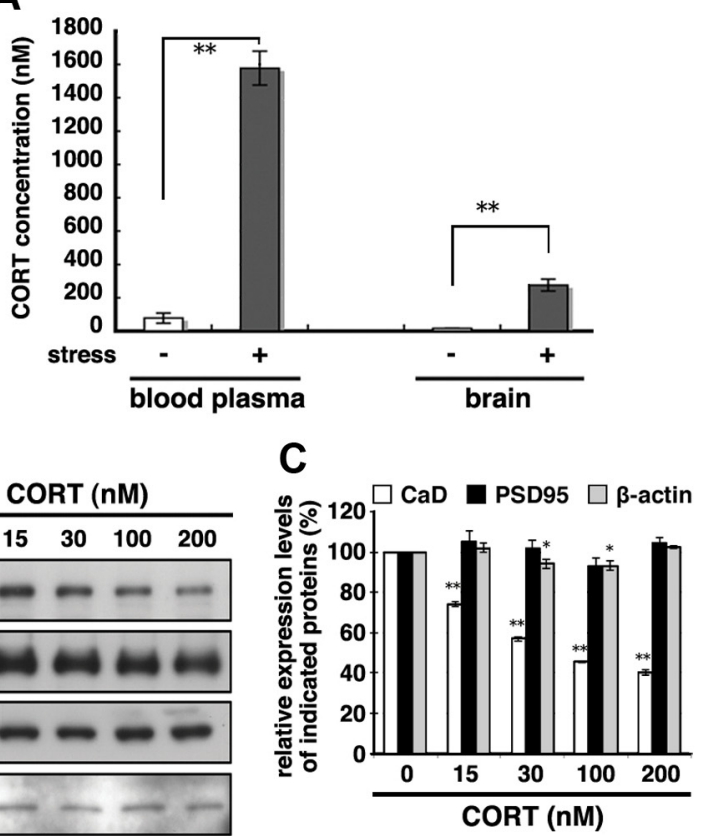

$\mathbf{E}$

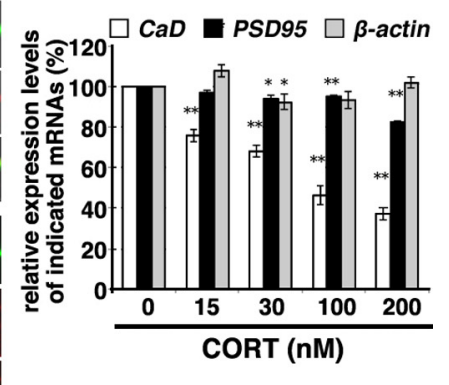

Figure 5. CORT decreases CaD expression. A, A graph of CORT concentrations in blood plasma and brain extract; stress-induced CORT concentrations were measured shortly after rats received four uncontrollable, inescapable foot shocks. Data are means $\pm S E$ red samples at 21-24 DIV. $\boldsymbol{C}$, Immunoblots shown in $\boldsymbol{B}$ were quantified by densitometry. Data are means \pm SE 0 nu CORT were fixed and stained with anti-GFP and anti-CaD antibodies (scale bar, 10 , on CaD, PSD-95, and $\beta$-actin mRNA levels, determined by RT-PCR. Data are means \pm SE of values from three independent experiments $\left({ }^{*} p<0.05,{ }^{* *} p<0.01\right)$.

moter contains two putative cis-elements: a serum response factor (SRF)-binding CArG-box and a positive glucocorticoid receptor (GR)-binding glucocorticoid-responsive element (GRE)-like sequence (Mayanagi et al., 2008). In hippocampal neurons, the positive GRE-like sequence was not involved in $\mathrm{CaD}$ promoter activity, whereas a CArG-box mutation reduced the $\mathrm{CaD}$ promoter basal activity; CORT treatment did not produce any additional effect on this activity (Fig. 6B). These results suggest that $\mathrm{CaD}$ transcription was promoted by the $\mathrm{SRF} / \mathrm{CArG}-\mathrm{box}$ and was reduced by CORT through inhibition of the SRF/CArG-boxdependent transcription.

CORT prevents spine development by downregulating CaD Consistent with the decrease in $\mathrm{CaD}$ and F-actin contents, CORT treatment resulted in smaller spine heads and a larger proportion of thin spines. At CORT levels of 100-200 nM, as found in the rat 

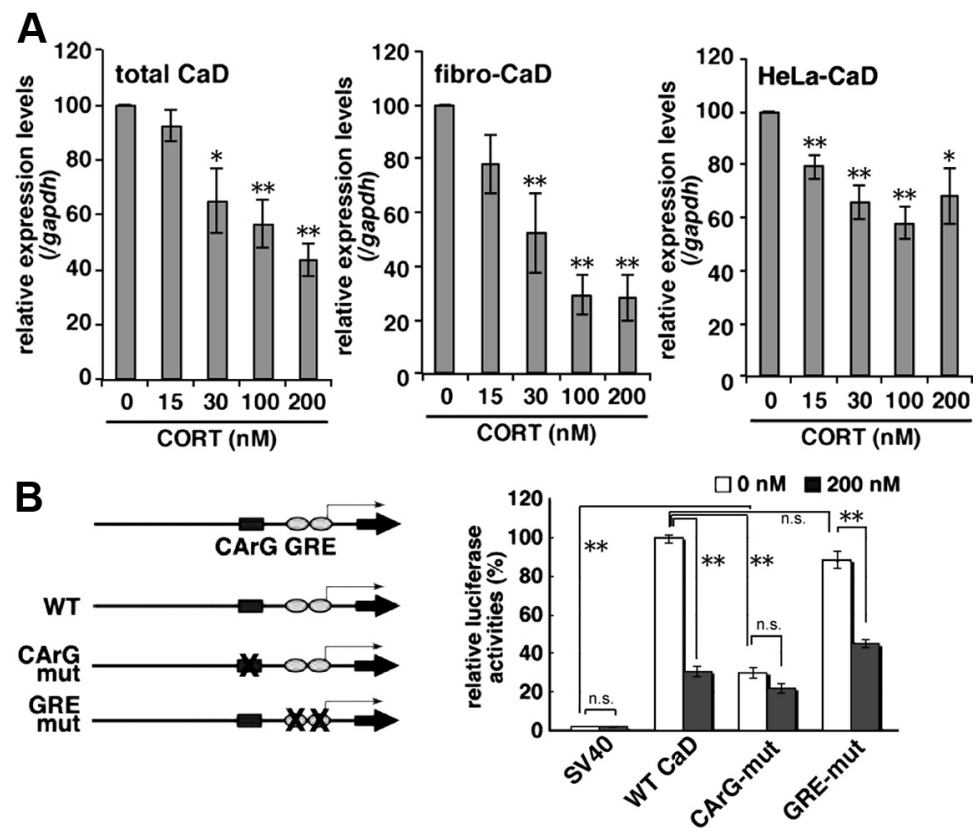

Figure 6. CORT effects on CaD isoform levels. $A$, Hippocampal neurons were cultured with CORT at the indicated concentrations. Total RNA was extracted, and RT-PCR was performed using specific primers for total $\mathrm{CaD}$, fibro-type $\mathrm{CaD}$, and HeLa-type $\mathrm{CaD}$. Data are means \pm SE of values from five independent experiments $\left({ }^{*} p<0.05,{ }^{* *} p<0.01\right)$. $\boldsymbol{B}$, A schematic diagram of mutation constructs of the CArG-box or GRE-like sequences in the rat fibro-type CaD promoter. Reporter assays were performed in hippocampal neurons (21-23 DIV) cultured with 0 or $200 \mathrm{~nm}$ CORT using the indicated promoter constructs. Reporter plasmid containing SV40 promoter (pGL3promoter) was used as a control. Data are means \pm SE of values from three independent experiments $\left({ }^{* *} p<0.01\right)$.

brain under stress conditions, thin spines predominated with a corresponding decrease in the number of mushroom spines (Fig. $7 A-C)$. FRAP assay demonstrated that CORT increased actin turnover rate (Fig. 7D). The time constants for actin turnover were $19.1 \pm 0.7 \mathrm{~s}$ in the neurons cultured with $200 \mathrm{nM}$ CORT compared with $28.6 \pm 0.6 \mathrm{~s}$ in those cultured without CORT $(p<0.01)$. LifeAct assay also showed that CORT reduced the spine F-actin stability (Fig. $7 E$ ). As many actin-regulating proteins have been reported to modify spine shape, head size, and function (Lisman, 2003), we used RT-PCR to examine the expression of 25 actin-regulating genes. While CORT treatment affected the expression of some of these genes to a degree (data not shown), only $\mathrm{CaD}$ expression was markedly decreased (Fig. 5B-E). To confirm the importance of $\mathrm{CaD}$ reduction in the detrimental effect of CORT on dendritic spine development, we overexpressed $\mathrm{CaD}$ under high CORT concentrations. Exogenous $\mathrm{CaD}$ enlarged the spine-head size and promoted mushroom-shape spines even in the presence of 200 nM CORT (Fig. $8 A-C$ ). The spine F-actin stability that was reduced by CORT treatment was recovered by exogenous $\mathrm{CaD}$ (Fig. $8 D, E$ ). Both FRAP and LifeAct assays showed that exogenous $\mathrm{CaD}$ stabilized F-actin even under $200 \mathrm{~nm}$ CORT treatment [time constants in FRAP for CORT-treated spines: $17.8 \pm 0.9 \mathrm{~s}$ vs for GFP-CaD-transfected CORT-treated spines: $28.0 \pm 0.8 \mathrm{~s}(p<0.01)]$. These results suggest that $\mathrm{CaD}$ is a critical downstream player in the detrimental effects of CORT on actin cytoskeleton-dependent development of dendritic spines.

\section{Discussion}

In this study, we demonstrated that $\mathrm{CaD}$ modulates the spinehead size in cultured hippocampal neurons by regulating F-actin dynamics. $\mathrm{CaD}$ inhibits actin-myosin interactions and potently stabilizes F-actin (Sobue and Sellers, 1991; Mayanagi and Sobue, 2011). In hippocampal neurons, $\mathrm{CaD}$ was accumulated in spines via an interaction with $\mathrm{F}$-actin (Fig. $1 B$ ), indicating that the function of $\mathrm{CaD}$ in spines depends on actin. Both fulllength $\mathrm{CaD}$ and the $\mathrm{CaD} \mathrm{C}$-terminal fragment stabilized F-actin and increased the spine-head size (Fig. 2). Chemical LTP induction requires reorganization of the actin cytoskeleton, which shifts the G/Factin ratio toward stable $\mathrm{F}$-actin and increases the spine-head size ( $\mathrm{Gu}$ et al., 2010). CaD was accumulated in spine heads in response to cLTP and stabilized the actin dynamics (Fig. 4). These results indicated that $\mathrm{CaD}$ was integral to this actin reorganization in spines (Figs. 2-4). Thus, F-actin dynamics regulated by $\mathrm{CaD}$ is critically involved in spine development and plasticity. Because $\mathrm{CaD}$ localization at spine heads also depends on F-actin, cooperative bidirectional interactions between $\mathrm{CaD}$ and F-actin may be ultimately required for regulating spine stability.

Neuronal architectures such as dendritic arborization and synaptic structure have plasticity in response to various factors, and this structural remodeling occurs throughout the lifespan (Dumitriu et al., 2010; McEwen, 2010; Liston and Gan,

2011; Bloss et al., 2011). These alterations correlate with cognitive and behavioral functions (Dumitriu et al., 2010; Bloss et al., 2011). Although transient stress exposure evokes adaptive responses of the hypothalamus-pituitary-adrenal (HPA)-axis, repeated or chronic stress causes the hyperactivation of this cascade. GCs have been intensely studied as a principal stress mediator. Exposure to high GCs triggers adverse effects on synapse formation, dendritic arborization, and hippocampal volume (Watanabe et al., 1992; Magariños et al., 1996; McEwen, 1999, 2005; Wellman, 2001; Radley et al., 2004; Radley et al., 2006; Liston and Gan, 2011). However, the molecular mechanisms are not fully understood. To examine the effects of GCs on cultured hippocampal neurons, we first measured the actual CORT concentrations in the blood plasma and brain of rats with or without foot-shock stress. Unexpectedly, the CORT concentrations in the brain were fivefold lower than those in the blood plasma, but they increased 16-fold after foot-shock stress (Fig. $5 A$ ). To test the effect of CORT levels on hippocampal neurons, we used a culture medium with a CORT-free B27 supplement and added CORT to defined concentrations. Using this culture system, we demonstrated that CORT at physiological concentrations inhibited dendritic spine development (Fig. 7A-C). F-actin dynamics are strongly linked to spine shape, motility, and plasticity (Hotulainen and Hoogenraad, 2010). CORT treatment destabilized the spine F-actin dynamics, changing the spine morphology from a mature mushroom shape to premature, thin, and filopodial shapes, as well as decreasing the spine-head size (Fig. 7). These data provide a novel insight regarding CORTinduced spine shrinkage as well as suppression of synapse formation and maturation. Previous in vivo histological studies have shown that chronic stress exposure decrease the number of dendritic spines (Radley et al., 2006). In addition, Liston and Gan 
(2011) have recently reported that in the living cortex, CORT exposure leads to the loss of stable, mature spines with increasing filopodial and thin spines and spine turnover rates. The mechanism revealed by our study would serve a molecular basis to explain those events concerning spine remodeling through a perspective of F-actin dynamics.

CORT downregulated the CaD expression levels in hippocampal neurons and suppressed the fibro-type CaD promoter's SRF/CArG-box-dependent activity (Fig. 6). In this regard, activated GR inhibits SRF activity in human prostate adenocarcinoma cells (Yemelyanov et al., 2007). We previously demonstrated that GC upregulates $\mathrm{CaD}$ expression via positive GRE-like sequences in both human lung cancer cells and cortical neural progenitor cells (Mayanagi et al., 2008; Fukumoto et al., 2009). However, in hippocampal neurons the GRE-like sequences did not function for the negative regulation of $\mathrm{CaD}$ transcription and the direct contribution of GR via the GRE-like sequences, therefore, seems to be less significant. These different responses against GC may be due to cellular contexts. This mechanism is required for future analysis.

As documented here, $\mathrm{CaD}$ plays an important role in synaptic plasticity as well, because $\mathrm{CaD}$ is required for synaptic remodeling accompanied with chemically induced LTP. CaD plays also a role as a crucial downstream target in the stress/ GC-induced effects on dendritic spine development. The abnormality of spine structure is considered to be associated with pathological dysfunction of the synapse. Indeed, psychiatric and neurologic disorders such as depression, schizophrenia, and Alzheimer's disease are characterized by pathologically altered spines (van Spronsen and Hoogenraad, 2010; Penzes et al., 2011). Thus, CaD may be involved in the stress-induced psychiatric disorders. Since spines and dendrites in young or aged rat brains are reported to exhibit different responsiveness against chronic stress (Dumitriu et al., 2010; Bloss et al., 2011), future study is required for this mechanism involving $\mathrm{CaD}$. Our present study provides new insight into the molecular basis of stress/GC-induced synaptic remodeling, and detailed investigation of the relationship between stress/GCs and CaD should improve our understanding of stress-induced neuronal plasticity and the related psychiatric disorders.

\section{References}

Becker JB, Monteggia LM, Perrot-Sinal TS, Romeo RD, Taylor JR, Yehuda R, Bale TL (2007) Stress and disease: is being female a predisposing factor? J Neurosci 27:11851-11855.

Bloss EB, Janssen WG, Ohm DT, Yuk FJ, Wadsworth S, Saardi KM, McEwen BS, Morrison JH (2011) Evidence for reduced experience-dependent dendritic spine plasticity in the aging prefrontal cortex. J Neurosci 31: 7831-7839.

Dumitriu D, Hao J, Hara Y, Kaufmann J, Janssen WG, Lou W, Rapp PR,

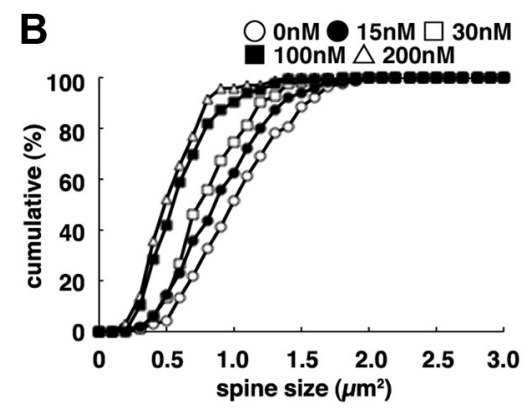

OnM 1 15nM $\square 30 \mathrm{nM}$ N 100nM $\square 200 \mathrm{nM}$
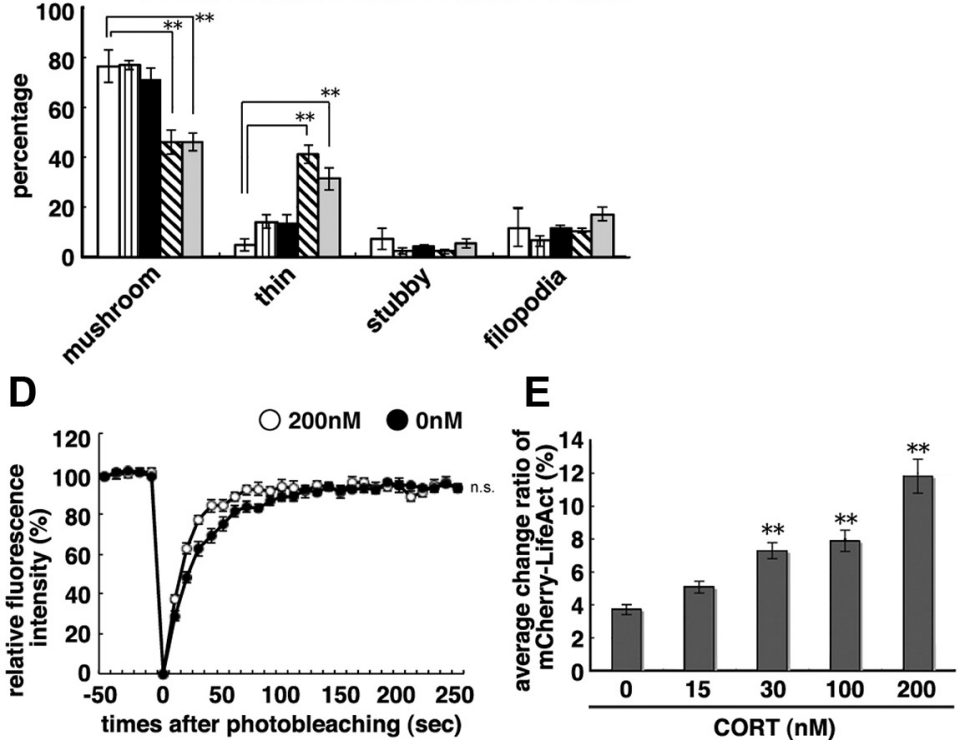

Figure 7. CORT effects on spine morphology and dynamics. $\boldsymbol{A}$, Spine morphology in GFP-transfected hippocampal neurons cultured with $0-200 \mathrm{~nm} \mathrm{CORT} \mathrm{(scale} \mathrm{bar,} 10 \mu \mathrm{m}$ ); (B) the graph shows spine sizes and their cumulative distributions. $\boldsymbol{C}$, Dendritic 200 n CORT. Davescence recovery of mCherry- $\beta$-actin over time after photobleaching, measured in neurons cultured with 0 or . Data are means \pm SE of values from 12 cells. $\boldsymbol{E}$, CORT dose-dependent effects on spine F-actin dynamics were measured using mCherry-LifeAct. The graph shows the change rates of mCherry-LifeAct fluorescence intensity in spines. Data are means $\pm S E$ of values from at least 27 spines $\left({ }^{* *} p<0.01\right)$.

Morrison JH (2010) Selective changes in thin density and morphology in monkey prefrontal cortex correlate with aging-related cognitive impairment. J Neurosci 30:7507-7515.

Fischer M, Kaech S, Knutti D, Matus A (1998) Rapid actin-based plasticity in Dendritic spines. Neuron 20;847-854.

Fischer M, Kaech S, Wagner U, Brinkhaus H, Matus A (2000) Glutamate receptors regulate actin-based plasticity in dendritic spines. Nat Neurosci 3: 887-894.

Fukumoto K, Morita T, Mayanagi T, Tanokashira D, Yoshida T, Sakai A, Sobue K (2009) Detrimental effects of glucocorticoids on neuronal migration during brain development. Mol Psychiatry 14:1119-1131.

Gu J, Lee CW, Fan Y, Komlos D, Tang X, Sun C, Yu K, Hartzell HC, Chen G, Bamburg JR, Zheng JQ (2010) ADF/cofilin-mediated actin dynamics regulate AMPA receptor trafficking during synaptic plasticity. Nat Neurosci 13:1208-1215.

Hotulainen P, Hoogenraad CC (2010) Actin in dendritic spines: connecting dynamics to function. J Cell Biol 189:619-629.

Jiang M, Chen G (2006) High $\mathrm{Ca}^{2+}$-phosphate transfection efficiency in low-density neuronal cultures. Nat Protoc 1:695-700.

Korkotian E, Segal M (2001) Regulation of dendritic spine motility in cultured hippocampal neurons. J Neurosci 21:6115-6124.

Lisman J (2003) Actin's actions in LTP-induced synapse growth. Neuron 38:361-362.

Liston C, Gan WB (2011) Glucocorticoids are critical regulators of dendritic spine development and plasticity in vivo. Proc Natl Acad Sci U S A 108: 16074-16079. 
A

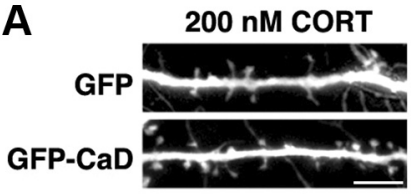

B
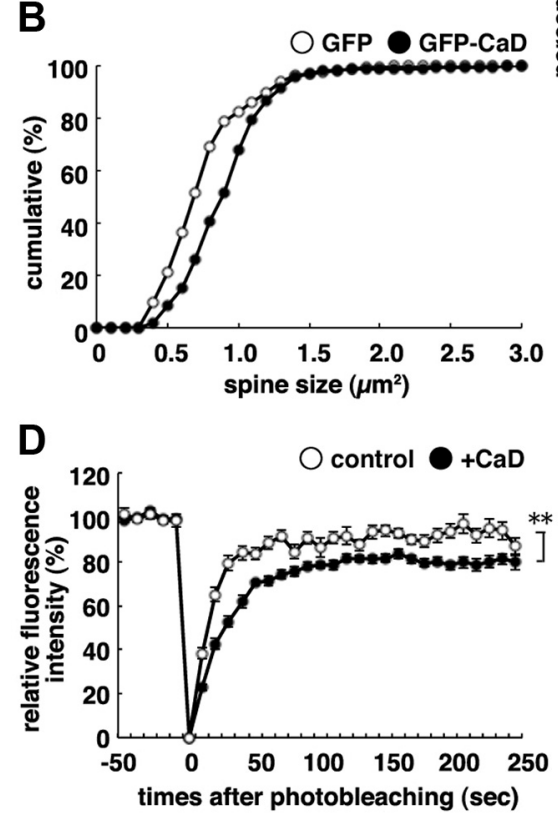

C

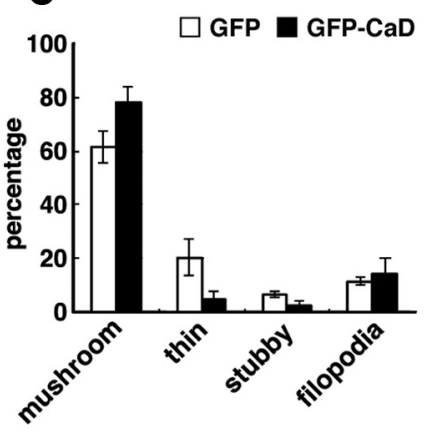

E

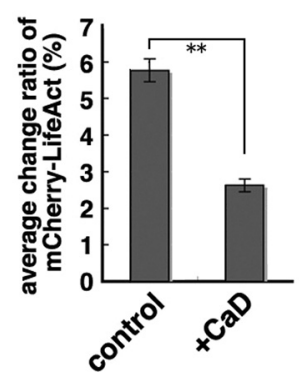

Figure 8. CaD recovers the CORT-induced dendritic spine changes. $A$, Spine morphology in neurons cultured with $200 \mathrm{~nm}$ CORT and transfected with GFP or GFP-CaD (scale bar, $10 \mu \mathrm{m})$; (B) the graph shows spine sizes and their cumulative distributions. $\boldsymbol{C}$, Dendritic protrusions, classified by morphology: mushroom, thin, stubby, and filopodial. Data are means \pm SE of values from at least 1214 spines. $\boldsymbol{D}$, The fluorescence recovery of $\mathrm{mCherry}-\beta$-actin over time after photobleaching, measured in neurons cultured with $200 \mathrm{~nm}$ CORT and transfected with a mock vector (control) or myc-tagged-CaD. Data are means \pm SE of values from 16 cells $\left(*^{* *}<0.01\right) . E$, Spine F-actin dynamics in neurons cultured with $200 \mathrm{~nm}$ CORT and transfected by mock vector (control) or myc-tagged-CaD were measured using mCherry-LifeAct. The graph shows the change rates of mCherry-LifeAct fluorescence intensity in spines. Data are means \pm SE of values from at least 21 spines $\left({ }^{* *} p<0.01\right)$

Magariños AM, McEwen BS, Flügge G, Fuchs E (1996) Chronic psychosocial stress causes apical dendritic atrophy of hippocampal CA3 pyramidal neurons in subordinate tree shrews. J Neurosci 16:3534-3540.

Mayanagi T, Sobue K (2011) Diversification of caldesmon-linked actin cytoskeleton in cell motility. Cell Adh Migr 5:150-159.

Mayanagi T, Morita T, Hayashi K, Fukumoto K, Sobue K (2008) Glucocorticoid receptor-mediated expression of caldesmon regulates cell migration via the reorganization of the actin cytoskeleton. J Biol Chem 283:31183-31196.

McEwen BS (1999) Stress and hippocampal plasticity. Annu Rev Neurosci 22:105-122.

McEwen BS (2005) Glucocorticoids, depression, and mood disorders: structural remodeling in the brain. Metabolism 54:20-23.

McEwen BS (2010) Stress, sex, and neural adaptation to a changing environment: mechanisms of neuronal remodeling. Ann NY Acad Sci 1204:E38-E59.

Modi N, Lewis H, Al-Naqeeb N, Ajayi-Obe M, Doré CJ, Rutherford M (2001) The effects of repeated antenatal glucocorticoid therapy on the developing brain. Pediatr Res 50:581-585.
Morita T, Mayanagi T, Sobue K (2012) Caldesmon regulates axon extension through interaction with Myosin II. J Biol Chem 287:3349-3356.

Otmakhov N, Khibnik L, Otmakhova N, Carpenter S, Riahi S, Asrican B, Lisman J (2004) Forskolin-induced LTP in the CA1 hippocampal region is NMDA receptor dependent. J Neurophysiol 91:1955-1962.

Penzes P, Cahill ME, Jones KA, VanLeeuwen JE, Woolfrey KM (2011) Dendritic spine pathology in neuropsychiatric disorders. Nat Neurosci 14:285-293.

Phillips NK, Hammen CL, Brennan PA, Najman JM, Bor W (2005) Early adversity and the prospective prediction of depressive and anxiety disorders in adolescents. J Abnorm Child Psychol 33:13-24.

Radley JJ, Sisti HM, Hao J, Rocher AB, McCall T, Hof PR, McEwen BS, Morrison JH (2004) Chronic behavioral stress induces apical dendritic reorganization in pyramidal neurons of the medial prefrontal cortex. Neuroscience 125:1-6.

Radley JJ, Rocher AB, Miller M, Janssen WG, Liston $\mathrm{C}$, Hof PR, McEwen BS, Morrison JH (2006) Repeated stress induces dendritic spine loss in the rat medial prefrontal cortex. Cereb Cortex 16:313-320.

Sarmiere PD, Bamburg JR (2004) Regulation of the neuronal actin cytoskeleton by $\mathrm{ADF} / \mathrm{cofi}$ lin. J Neurobiol 58:103-117.

Sobue K, Sellers JR (1991) Caldesmon, a novel regulatory protein in smooth muscle and nonmuscle actomyosin systems. J Biol Chem 266:12115-12118.

Star EN, Kwiatkowski DJ, Murthy VN (2002) Rapid turnover of actin in dendritic spines and its regulation by activity. Nat Neurosci 5:239-246.

van Spronsen M, Hoogenraad CC (2010) Synapse pathology in psychiatric and neurologic disease. Curr Neurol Neurosci Rep 10:207-214.

von Bohlen Und Halbach O (2009) Structure and function of dendritic spines within the hippocampus. Ann Anat 191:518-531.

Watanabe Y, Gould E, McEwen BS (1992) Stress induces atrophy of apical dendrites of hippocampal CA3 pyramidal neurons. Brain Res 588:341-345.

Weinstock M (2008) The long-term behavioural consequences of prenatal stress. Neurosci Biobehav Rev 32:1073-1086.

Wellman CL (2001) Dendritic reorganization in pyramidal neurons in medial prefrontal cortex after chronic corticosterone administration. J Neurobiol 49:245-253.

Yano H, Hayashi K, Haruna M, Sobue K (1994) Identification of two distinct promoters in the chicken caldesmon gene. Biochem Biophys Res Commun 201:618-626.

Yemelyanov A, Czwornog J, Chebotaev D, Karseladze A, Kulevitch E, Yang X, Budunova I (2007) Tumor suppressor activity of glucocorticoid receptor in the prostate. Oncogene 26:1885-1896. 